\title{
MacProbe: A Macintosh-based experimenter's workstation for the cognitive sciences
}

\author{
STEVEN M. J. HUNT \\ University of California, Los Angeles, California
}

\begin{abstract}
MacProbe is a program that turns an Apple Macintosh with a 68020 processor or greater and a floating point unit into an experimenter's workstation for implementing a large class of experimental paradigms characteristic of the interdisciplinary fields constituting the cognitive sciences. The core of MacProbe is a structured, interpreted programming language with over 200 high-level commands that provide support for all facets of experimentation from design and presentation of visual and auditory probes, to real-time experiment control, to the analysis and management of experimental data and the presentation of results. The programming language is supplemented by a graphical user interface for such tasks as text and waveform editing and determining the placement of visual probes.
\end{abstract}

Personal computers have become an increasingly important tool for performing experiments in the cognitive sciences. As the area evolves, the demands placed upon these computers can be expected to increase even further, requiring systems that can present both visual and auditory probes with a high degree of accuracy and simultaneously acquire a variety of types of data, from simple manual or verbal responses to physiological waveforms such as eye movements, evoked potentials, or even action potentials. Further demands will also be placed on the user interface, requiring it to support a wider range of paradigms and to provide a more powerful program development environment so that computer-based experiments can be quickly implemented with a minimum amount of effort and a minimum requirement for specialized programming or engineering skills, despite their ever increasing sophistication.

MacProbe represents a first attempt at creating such an interdisciplinary tool for computer-assisted experimentation. The model for MacProbe is that of a computer workstation similar to those used in fields such as mechanical engineering, architecture, and desktop publishing: a system of integrated, easy-to-use, general-purpose tools accessed through a window-based, object-oriented graphical user interface. The choice of the Macintosh II platform was influenced by several considerations: (1) the wealth of high-quality hardware and software available for the Macintosh for the creation and presentation of visual and auditory probes; (2) the high degree of interconnectivity of applications (thanks to the clipboard and a standardized user interface); (3) the 32-bit linear address space, allowing large numbers of visual and auditory probes to

Correspondence should be addressed to S. M. J. Hunt, 21002 Bandera St., Woodland Hills, CA 91364.

Note: The author has a direct financial interest in MacProbe.-Editor be preloaded and straightforward operation on data structures larger than $64 \mathrm{~K}$; (4) the ease of use provided by its intuitive, object-oriented graphical user interface; and (5) affordability for those in the academic research community.

\section{Command Language}

The heart of MacProbe is a C-like, interpreted programming language. In addition to the typical program control and user I/O commands, it provides over 200 high-level commands for digital and analog signal $\mathrm{I} / \mathrm{O}$, visual and auditory probe presentation, and data management and analysis. A sample of the command set is provided in the Appendix. The command set has been engineered to give the experiment designer access to the full capabilities of the Macintosh II family hardware and graphical user interface while hiding, as much as possible, the complex internal details of the computer and of the Macintosh toolbox.

The commands operate on variables that may be scalars or arrays of one or two dimensions. The variables may have any of the standard numeric formats from byte-sized integers to 12-byte double-precision floatingpoint numbers. They may also be null byte terminated strings, string arrays, or character arrays. Array lengths are limited only by available RAM, and space for the arrays is allocated dynamically. On a IIci, for example, this would allow a maximum of nearly $32 \mathrm{Mb}$ of array data.

All the commands that operate on arrays are completely general purpose. That is, they make no unnecessary assumptions about the nature of the data they operate on. Thus, for example, the same command that is used to sort an array of lexical tokens to be presented as probes can also be used to sort the values of a dependent or independent variable as a function of trial number, or the names of data files to be analyzed, or even the intensities of individual pixels in an image to be used as a probe. 


\section{User Interface}

When MacProbe is launched, it automatically opens a text window that can be used as a worksheet for entering commands and displaying the results of operations. The user can open as many of these text windows as desired and transfer data between them via the clipboard. Typing (return at the end of a line in one of these windows simply inserts a return in the text, whereas typing (enter) treats the line as a command and executes it. Multiple command lines can be executed by simply selecting them with the mouse and pressing the (enter) key.

A program to perform a task, such as experiment presentation, is built by combining a number of these commands in one of the text windows. If a window containing commands is given a name ending in ".mc" it officially becomes a script window and can be executed as a unit by selecting the window with the mouse and then typing (command)-R. Storing the contents of this window to the disk defines a script file, which can be run at any time by choosing the Select function on the scripts menu or by double-clicking on the script file from the finder.

Scripts can also be executed by simply entering the name of the script as if it were a MacProbe command. If a command is issued in MacProbe but is not recognized, MacProbe will search in a predefined folder for a script file with the same name as the command and if the file is found, it will execute that script, effectively allowing the script designer to add new commands at will. These commands can also be added to the main menu bar.

In addition to text windows, MacProbe provides a mouse-driven, graphical user interface for such tasks as setting up visual presentations and editing waveforms. In many cases, the graphical operations can also be performed by using commands, so that the user can choose the most efficient method of accomplishing the task at hand.

\section{End User Interface}

A graphical user interface is convenient not only during the script design phase but also during script execution. For example, in a script that performs an experiment presentation sequence, the user may wish to be able to interactively set parameters that control the experiment presentation or to selectively initiate individual components of the sequence using the mouse (e.g., practice vs. test). To facilitate this, MacProbe provides commands that enable a script to create "control panels," which appear on the screen when the script is run. These panels are similar to Macintosh dialogs and can contain the familiar types of Macintosh dialog items: push buttons, check boxes, pop-up menus, lists, editable and static text items, and icon buttons. Additionally, they can contain polygraph style plots, $\mathrm{X} / \mathrm{Y}$ style plots, bar graph plots, and/or arbitrary images generated by using the picture command.

Each control in a panel can be attached to a subprocedure within the script that creates it. Subsequently, when that control is activated (for example, by pressing a button or clicking in a picture item), the corresponding script procedure is automatically executed and the state of the control (e.g., the item selected from a list or menu) is transmitted to the subprocedure. In this way, simple object-oriented scripts can be written to run experiments that provide a graphical "front end" for the user.

\section{Interpreted Versus Compiled Languages}

The choice of an interpreted instead of a compiled command language was based on two principal considerations. First, an interpreted language provides a simplicity of operation that makes the task of setting up experiments easier for those without extensive programming experience. With this approach, no header files, no object or executable files, and no multipass or multiphase operations are required during program development (such as editing, then compiling, then linking, etc.). Instead, programming is reduced to cutting and pasting text between text windows or files.

Second, an interpreted language provides a high degree of interactivity between the computer and the script designer, which greatly facilitates the kind of trial-anderror prototyping and fine tuning that characterizes experimentation. For example, while one is running a script in MacProbe, if a change is desired, typing (escape) instantly returns the user to the script window. After changing the appropriate command lines, choosing Run from the Scripts menu (or typing (command)$R$ ) runs the modified script immediately, allowing rapid incremental development of testing paradigms. Multiple script windows can be opened at the same time, and any one can be run by clicking in its window and typing (command)-R. If an error occurs, choosing Show Error from the Scripts menu (or typing (command)-E) instantly selects the script window and positions the cursor at the offending line. There is also a built-in script debugging facility, which can be used to step through a script, place breakpoints, and examine the value of variables. The script writer may also place commands in the script to output data to a text window or file for debugging purposes.

The principal disadvantage of an interpreted language is its execution speed. The MacProbe command language has been highly optimized so that script execution time, while not as fast as compiled $\mathrm{C}$ code, is more than adequate for most experimental paradigms and data analysis applications. The speed-flexibility tradeoff of interpreted code is further compensated for by the many high-level commands built into MacProbe that operate on entire arrays at once rather than element by element. For example, there are high-level commands to sort one or multiple arrays, to randomize element order in an array, to compute the mean or median of an array, to read or write multiple arrays from a file, to search an array for a specified value, or to replace every occurrence of a specified value with another. Speed is also gained by having the commands operate on data in RAM. 
For computing requirements that exceed the capabilities of MacProbe's interpreted language, a C interface kit is also available, which allows a programmer with an intermediate knowledge of $\mathrm{C}$ to create plug-in MacProbe commands using the Think $\mathrm{C}$ development system. In this way, special-purpose commands can be optimally coded in $\mathrm{C}$ or assembler for speed after being tested and debugged by using the MacProbe command language and then ultimately called as $\mathrm{C}$ plug-in commands from the interpreted script. Once installed, these $\mathrm{C}$ plug-in commands look and operate just like regular MacProbe commands from the standpoint of the script designer.

An area in which both compiled and interpreted languages can run into trouble concerns probe presentation and response collection in which a precise timing sequence must be maintained. MacProbe deals with this problem by providing two special commands for realtime experiment presentation and control: psseq and rtp.

The command psseq allows the experiment designer to specify a sequence of events to occur, synchronized to the vertical retrace of the screen on which images are to be presented. These events can include displaying images, collecting responses from the keyboard or other devices, playing sounds or alerting tones through the internal sound hardware or a DigiDesign AudioMedia board. MacProbe compiles these commands into a state table, simultaneously optimizing the format of any images to be displayed to ensure the fastest possible screen drawing. It then initiates the sequence. In this way, precise timing and high-speed operation is achieved, despite the fact that the sequence is actually specified in an interpreted command language.

Since the ability to perform a given presentation sequence will ultimately depend on the size and complexity of the images to be presented, the speed of the CPU, and video RAM access time, all of which are factors difficult to predict in advance, the psseq command also includes a debug option that will automatically construct a table of the actual event execution times versus the requested execution times. The reference times can be derived either from the Macintosh VIA timer (using the extended time manager under System 7 with 1 -msec accuracy or the original time manager under System 6) or a National Instruments Lab-NB board which has onboard 8253 timers with $1-\mu$ sec accuracy. After the sequence is complete, the experiment designer can examine the table to discover whether or not there is adequate processing speed to actually perform the sequence as specified and can make adjustments accordingly.

For presentation paradigms that cannot be described by a linear sequence of events (e.g., real-time adaptive testing in which the sequence of events during a trial is contingent upon one or more of the inputs measured during that trial), there is the rtp command. This command has its own subcommand language, which resembles an assembly language. The subcommands are assembled in advance before beginning the process. It employs a double-queuing system that allows the user to set up mul- tiple processes to be performed, nonpreemptively, in a pseudoparallel manner concurrent with data acquisition from a National Instruments Lab-NB and/or NB-DIO24 board if desired. There is approximately a three-toone ratio of execution speed between rtp commands and standard, nonoptimized, $680 \times 0$ assembly language code. Furthermore, as is the case for the main command language, the rtp language is highly optimized and contains many high-level instructions, so that, for example, there are single rtp commands to display an image on the screen or read the mouse coordinates or branch, depending upon whether or not an input value is within a specified range.

\section{Visual Probe Generation}

MacProbe supports display of the contents of any PICT or TIFF file on any monitor or combination of monitors supported by the Macintosh II. In this way, the user can take advantage of the wealth of hardware and software available on the Macintosh for digitizing, drawing, and editing images. Images used as probes can be anything from monochrome line art to 24-bit color pixel maps.

MacProbe also has a high-level command (defimage) containing over 100 turtle graphics subcommands, which can be used to define images algorithmically. These images can be a mixture of line segments, ovals, rectangles, regions, text, and pixel maps derived from PICT or TIFF files. The resulting images can also be exported to other programs for further refinement.

For more complex visual probes, MacProbe provides commands that can transfer pixel maps and color tables to and from numeric arrays. This enables the experiment designer to algorithmically create pixel maps or to modify, digitally filter, or alter the colors or intensities of individual pixels in an existing pixel map.

\section{Visual Probe Display}

Visual probe display in MacProbe typically begins by specifying the monitor to be used (if more than one is attached to the computer), the background color, the color palette (if it is an indexed display), and a unit of measurement for indicating locations on the monitor at which images are to be presented. A visual presentation setup facility is built into MacProbe, which, when invoked, replaces the desktop with a calibrated grid in the desired measurement units-which can be pixels, inches, centimeters, or degrees of visual angle (based on a specified viewing distance). The user can then create and position rectangles called presentation windows on the screen and give them names. These windows can subsequently be referred to by name in the script when one is specifying where to display an image. Selecting the Save Settings option from the presentation screen setup dialog generates all of the MacProbe commands needed to reproduce the desired presentation screen parameters and presentation windows and places these commands in the worksheet, from which they can be copied into a script window. 
Alternatively, the experiment designer can simply generate the reference points algorithmically or just specify the location where images are to be displayed directly in screen coordinates in any of the supported units of measurement. The use of presentation windows, however, allows automatic cropping or resizing of images (with or without aspect ratio preservation) to a fixed rectangular area in order to standardize their visual field extent.

To display images, there is a comprehensive set of ps (presentation screen) commands, including the psseq command described earlier. These commands treat images as individual objects. The objects can be displayed and removed independently and can overlap. All image presentation event times are specified in terms of video frames rather than milliseconds, so that the quantization of exposure times imposed by the raster display is made explicit.

In addition to commands for displaying the contents of PICT or TIFF files, there are commands for displaying text in any available font size and style and for displaying simple geometric shapes such as lines, rectangles, and ovals in outline or solid form. The text can be displayed vertically, horizontally, or diagonally, with complete control over spacing and leading. The fonts can be PostScript, TrueType or bit-mapped.

There are also commands for performing common forms of image animation, such as scrolling, wiping, fading, and manipulating color lookup tables, and for collecting responses from the subject by using a mouse or another ADB device such as choosing an object from a matrix, specifying a point on a rating scale, dragging or resizing lines or rectangular areas, or freehand drawing. Finally, there is a psmovie command, which can be used to display QuickTime movies or animation sequences on the screen.

For psychophysical paradigms requiring precise control of display contrast, MacProbe provides commands for loading the display's gamma correction table from within a script and for loading and using custom color lookup tables when presenting images. These features, when used along with a device such as the video attenuator described by Pelli and Zhang (Pelli \& Zhang, 1991), permit very accurate control of contrast on monitors with standard 8-bit display cards.

\section{Auditory Probes}

MacProbe provides a built-in digital scope for recording sounds using the internal sound chip, a MacRecorder, or the National Instruments Lab-NB board. MacProbe can also play sounds through a DigiDesign AudioMedia Board, which provides 16-bit, $44.1-\mathrm{kHz}$ stereo sampling and playback. The digital scope has a complete set of editing commands and can operate on up to four channels, so that waveforms can be easily cut and spliced. Since stereo recording and playback is supported, dichotic auditory probes can also be created. These can be combined with visual presentations by using the psseq or the rtp command.
There are also facilities for ramping the leading and trailing edges of waveforms to avoid clicks and for mixing two waveforms. Auditory waveforms can also be generated directly from numeric arrays if desired, and they can be edited as numeric arrays as well. The FFT and IFFT commands provided for signal processing, in particular, can be used to generate auditory waveforms quickly from specified spectral components.

\section{I/O Commands}

MacProbe provides a comprehensive set of commands for $\mathrm{I} / \mathrm{O}$ to and from various types of files, including ASCII text files, MacProbe native-format binary files (called bfiles), arbitrarily formatted binary files, or even Macintosh resources. The native binary format consists of one or more variable-length, variableformat records, each of which contains a set of scalar and/or array variables and a corresponding symbol table. Since a symbol table is stored with each record, the bfile formats are self-documenting, so that any MacProbe user can immediately read bfiles created by another user without requiring additional formatting information.

The raw data I/O commands support reading and writing arbitrarily formatted binary numeric data in both Motorola and Intel format, as well as strings written in C, Pascal, or FORTRAN formats, and can thus be used to access files created by other programs, even if they have been created on other platforms.

I/O can also be performed between scripts and text windows using the ew command. This command can be used to write programmable text editors for parsing or analyzing textual material. The text I/O commands support easy conversion of data to formats required by various standard statistical packages on both Macintoshand DOS/Windows-based platforms.

Other I/O commands support access to the serial interface for such purposes as controlling RS-232- or RS-422-based devices, talking to other computers in multiprocessor configurations, or telecommunications. There is also support for reading and writing MIDI data packets and recording analog and digital waveforms by using the National Instruments Lab-NB or NB-DIO-24 board. This enables use of MacProbe with physiological amplifiers and signal conditioners, eye trackers, and other forms of analog or digital instrumentation. The keyboard, mouse, and other ADB devices can also be accessed from within a script.

For recording event occurrence times (corresponding to switch presses, voice onset, etc.), MacProbe also supports a computer interface box designed by the author that uses its own embedded microcontroller to time stamp the events with better than .25-msec accuracy and then transmits the data to the Macintosh via a high-speed serial connection. This interface circumvents the problem of deriving manual response latencies from the Macintosh ADB devices, which presently have an inherent $11-\mathrm{msec}$ latency, and also ensures accurate response time recording independent of processor load. In 
situations where the 11-msec latency is acceptable, manual responses can also be derived from any ADB keyboard attached to the Macintosh.

\section{Data Management}

MacProbe contains an extensive set of high-level commands for performing data management on arrays, including sorting and randomizing elements, copying, appending, inserting, deleting, and shifting elements. In MacProbe, any set of two or more arrays can effectively constitute a database with element number becoming the record number and each array corresponding to a field in the database. For this purpose, there are high-level commands for performing queries on these databases and retrieving selected subsets of information as well as generating sorted indexes. An index can be generated, for example, in which each keyword corresponds to one combination of the levels of the independent and dependent variables.

\section{Data Analysis}

MacProbe includes high-level commands for performing basic arithmetic operations on arrays as well as summary statistics such as means, medians, standard deviations, and proportions. By using the database retrieval functions described above in conjunction with the summary statistics commands, MacProbe can generate a summary table of means, medians, or standard deviations for all relevant dependent and independent variable combinations with a single high-level command.

There are also commands for performing linear regression and computing histograms as well as built-in functions for computing probabilities and inverse probabilities from normal, Student's $t$, chi-square, binomial, and $F$ distributions.

\section{Waveform Analysis}

In addition to dealing with categorical data, MacProbe has an extensive set of commands for working with waveforms and discrete event data. These include com- putation of moving averages, differentiation, integration, automatic detection of peaks and jumps, digital filtering (both infinite impulse response and finite impulse response), and Fourier analysis.

Discrete time events are stored as standard numeric arrays in which each element represents the occurrence time of an event. For events which have a duration, adjacent pairs of array elements represent, respectively, the onset and offset time of the event. In this way, no special formats are needed for event data, and all of the regular array processing commands available in MacProbe can be used with event data in the same way they are used with waveforms and categorical data. Furthermore, many digital signal processing and summary statistics commands allow one to use the event arrays to specify which segments of an array to operate on. There is also a polygraph plot command that can display multiple waveforms and allow one to superimpose and edit events associated with the waveforms.

\section{Requirements}

MacProbe is designed to run on any Macintosh that has a floating-point coprocessor chip and a 68020 or greater CPU. It requires a minimum of $3 \mathrm{Mb}$ of RAM and runs under system 6.05 or later and is fully System 7 compatible.

\section{Availability}

MacProbe is available from Aristometrics Computer Systems (voice/fax, 818-883-1861; Internet, macprobe@) aristo.com). The price is $\$ 560$ for the Basic version with academic discount. The $\mathrm{C}$ plug-in development kit and the computer interface box are also available from the same source.

\section{REFERENCES}

Pelli, D. G., \& Zhang, L. (1991). Accurate control of contrast on microcomputer displays. Vision Research, 31, 1337-1345.

\section{APPENDIX \\ Sample of MacProbe Commands}

Programming
defvar
if
for
while
loop
case
Array Math
add
addc
abs
log
ptc
get mean
get stdev
get histo
get reg

define variable

conditional

for loop

while loop

loop through array

conditional blocks

add two arrays

add constant to array

absolute value of array

compute log of array

polar to cartesian

get mean of array elements

get standard deviation

compute histogram

compute linear regression 
Array Processing

append
copy
find
defindex
lsh
rand
sort
search
Event Processing
getpeaks
getjumps
evtoden
pevor
pevand

Miscellaneous

rtp

list ...

get ...

picture

pplot

xyplot

panel

dialog

verify

Visual Presentation
defpwin
psimage
psstring
psloadct
psmouse
psseq
psgetl
pspick
get imagepix
set imagepix
get imagect
set imagect
psslider
set pscreen
defimage
Sound Presentation
beep
definetune
play
Signal Processing
calibrate
deffilter
movav
deriv
integrate
demean

append two arrays copy from one array to other perform query define database index shift array elements left randomize element order sort one or more arrays perform bisection search

find peaks in waveform find jumps in waveform compute event density "or" events "and" events

real time programming list info in worksheet get various info create quickdraw picture create polygraph plot create $\mathrm{X} / \mathrm{Y}$ plot create a panel create a dialog verify operation of monitor etc

define a presentation window display image display string load color table perform mouse input perform presentation sequence get line of text from subject pick object from matrix convert pixmap to array convert array to pixmap convert color table to array convert array to color table perform rating scale input set presentation screen params define image algorithmically

produce alerting sound play sequence of notes play a sound file

calibrate a waveform define a digital filter perform moving average compute derivative compute integral subtract mean from waveform 
APPENDIX (Continued)

$\mathrm{I} / \mathrm{O}$

val

gval

valrdf

gvalrdf

bfile

gvalrstc

serial

midi

labnb

nbdio

adin

cib

output text to window or file input text from window or file write to raw binary data file read from raw binary data file $\mathrm{read} / \mathrm{write}$ to bfile

read from resource fork perform I/O to serial port perform I/O to MIDI devices perform I/O to Lab-NB

perform I/O to NB-DIO-24

perform $\mathrm{A} / \mathrm{D}$ conversion

access computer interface box

(Manuscript received November 3, 1992;

revision accepted for publication April 28, 1994.) 